\title{
Multi-Modes Approach to Modelling of Vortex-Induced Vibration
}

\author{
Ekaterina Pavlovskaia, Marko Keber, Andrey Postnikov, Kieran Reddington and Marian Wiercigroch \\ Centre for Applied Dynamics Research, School of Engineering, Aberdeen University, King's College, Aberdeen, AB24 3UE, \\ Scotland, UK
}

\begin{abstract}
In this work the fluid-structure interactions are considered by investigating a straight but slender pipe interacting with uniform water flow. Two configurations are studied, namely vertically and horizontally positioned pipes, which are modelled as an Euler-Bernoulli beam with flexural stiffness. Both pretension and length-wise mass distribution are considered. The structure is assumed to be moving only in the direction normal to flow (cross-flow motion) hence its in-line motion is neglected. The external fluid force acting on the structure is the result of the action of sectional vortex-induced drag and lift forces. Only mean drag force is considered, with time varying lift force modelled using a nonlinear oscillator equation of the Van der Pol type. The obtained coupled system of nonlinear partial differential equations is simplified employing Galerkin-type discretisation. The resulting ordinary differential equations are solved numerically providing multi-mode approximations of cross-flow displacement and non-dimensional lift coefficient. The comparison between the responses of vertical and horizontal structures shows that, as expected due to a balancing between pretension and weight, in general a higher amplitude of vibration is observed for the vertical configuration than in the same location along the pipe for the horizontal configuration in the lower part of the structure. However, lower amplitudes are obtained in the upper part of the pipe. The horizontal configuration solutions are identical in symmetrical locations along the pipe due to constant pretension. The influence of the wake equation coefficients and the fluid force coefficients on the response amplitudes has been also considered together with the length of the pipe and pretension level, and the appropriate response curves are included. Finally, for the higher mode approximations it has been shown that the vibrations level at lower frequencies is predicted reasonably well by retaining only a small subset of modes.
\end{abstract}

Vortex induced vibrations, Wake oscillator, Multi-mode approximation, Nonlinear analysis

\section{Introduction}

Slender marine structures such as piles, offshore risers, umbilicals, and others are often very sensitive to excitation induced by vortex shedding, which results in vibrations that in certain combinations of waves and current develop into a resonance phenomenon known as lock-in. This kind of vibrations can be destructive and may lead to structural collapse. Several decades of research have been devoted to exploring the complex nature of vortex-induced vibrations (VIVs), from purely fundamental studies of the phenomenon to attempts to develop a reliable toolkit that would allow predicting VIVs of marine structures with a desired precision. It should be noted, that VIVs can also develop in air, in particular in cable bridges, chimneys and tall buildings $[1,2]$ but the current study is focussed on this phenomenon in the water. 
The need of industry for an effective solution in this area led to the development of a number of software packages, such as SHEAR7 [3] or OrcaFlex [4], that utilize semi-empirical approaches to predict riser VIVs. One of the critical requirement for such tools is an acceptable simulation time. These tools normally use a coupled model of the fluid forces and the structural dynamics to simulate VIVs of a riser. The fluid force is usually modelled using semi-empirical equations such as Morison's equation. As a result, these tools are fast to run and they offer a cheaper and more practical alternative to carrying out physical experiments. However, the obvious drawbacks in this case are their limited accuracy and range of applicability.

When computational fluid dynamics became a more accessible way to model VIVs due to the increase of computational capabilities, a number of new modeling approaches were proposed, such as investigations and attempts to study the behavior of long flexible circular cylinders and full-scale riser systems with so-called strip theory $[5,6,7,8]$, which allows the observation of a multi-mode response of a flexible riser while implementing high aspect ratio 3D meshes [9]. Although this was definitely a step forward, the computation of a single CFD simulation still takes a significant amount of time for this approach to be effectively integrated and used by the industry. Some attempts of partial integrations have been made by OrcaFlex [4] by introducing the Vortex Tracking model, based on the underlying physical equations of boundary layer theory and the Navier-Stokes equations. However, there are some benefits in using full-scale CFD as a tool to calibrate existing semi-empirical reduced-order models, which will result in creation of a better, less computationally demanding models for VIVs prediction. Experimental studies on the force distribution along the axis of a flexible circular cylinder undergoing multi-mode VIVs have also been carried out, for example by Chaplin et al. [10] and Huera Huarte et al. [11], who in 2006 combined experimental data with the finite element method model of the riser. Recently, Franzini et al. $[12,13]$ obtained and used experimental data for determining hydrodynamic coefficients in the developed reduced order model of flexible cylinder subjected to prescribed top motions. Similar problem was considered by Mazzilli et. al [14] and non-linear modes based on Bessel functions were developed. These results could be also useful for the reduced-order models calibrations.

The analytical approach to VIVs is represented by a wide range of models, with some of them incorporating a Van der Pol type equation as the governing equation for the fluid force acting on the structure $[15,16]$. Since then, many modifications have been made to this particular approach, and the model itself became known as the wake oscillator model. The simplest wake oscillator model describes the interactions between a fluid flow and a rigid cylinder capable of moving in cross-flow direction only, with the system usually described by two coupled ordinary differential equations. One of the equations is the equation of motion of the structure, and the second equation is a semi-empirical description of the lift force generated by the passing fluid, a nonlinear self-excited fluid oscillator. In 1997 Balasubramanian and Skop [17] proposed the new model with two terms for the lift force. In previous models, a Van der Pol equation was employed as the governing equation for the entire lift force on the structure. The model introduced in [17] included a Van der Pol equation driven by the local transverse motion of the structure as a governing equation for one component of the fluctuating lift force and a so-called stall term which is linearly proportional to the local transverse velocity of the structure. Because of the stall term, an asymptotic, self-limiting structural response at zero structural damping was captured.

One of the most notable analysis in this class of low dimensional models in terms of the fundamental behaviour was made by Facchinetti et al. [18], where the transverse vibrations of one degree-of-freedom structures in stationary uniform flow were investigated. The analysis was undertaken by first estimating the values of all parameters based on the available experimental data on forced vortex shedding, and then solving numerically the fully coupled system. These results have been systematically compared with experimental data from the 
literature such as oscillation amplitude at lock-in, extension of lock-in and effective added mass. It was found that the acceleration coupling provided the best match for the experimental results qualitatively and, in some aspects, quantitatively. Later, Violette et al. [19] performed comparisons of the Facchinetti wake oscillator model of slender cylinder undergoing VIVs with DNS and experiments.

A coupled model incorporating two degrees-of-freedom motion of a rigid cylinder excited by harmonic fluid forces was proposed by Wang et al. [20] in 2003, who also considered an extension of this approach towards a slender structure by using Euler-Bernoulli beam theory. This work was later modified by Ge et al. [21], incorporating the Facchinetti wake oscillator [18]. In 2007 Furnes and Sorensen [22] developed a 3D time domain model called VIVITAS [22] with coupled nonlinear oscillators representing in-line and cross-flow fluid force components. Some empirical coefficients from the Facchinetti model [18] were tuned against the Marintek data [22]. The effect of variable tension on vortex induced vibration of vertical riser was studied in [23] where a finite element model of the structure was coupled to a wake-oscillator model of the van der Pol type. Overall, the wake oscillator models are not computationally demanding and hence can be implemented in various software packages for the design and analysis of offshore marine systems, and for instance, the Iwan and Blevins model [24] is already included in OrcaFlex [4].

Further investigations on the semi-empirical approach were presented in the work by Keber and Wiercigroch [25]. They investigated the effect of a weak structural nonlinearity on the dynamical behaviour of a vertical offshore riser undergoing VIVs. As offshore risers experience additional excitation from the flow of the fluid inside the pipe, this work considered the influence of internal flow and nonlinear coupling of axial and bending displacements. It was shown that the structural nonlinearity has a stiffening effect on the oscillation of the riser, which becomes more pronounced when the internal flow is incorporated into the model. Earlier Mazzilli et al. have conducted non-linear modal analysis of axially loaded beams in [26] exploring effects of structural nonlinearities. In 2009 Srinil, Wiercigroch and O'Brien [27] proposed a reduced-order model of the VIVs of a catenary riser, with a mathematical description of a multi-mode response of a slender structure combined with a wake oscillator model as the fluid force component; however, only very limited multi-mode analysis was presented. Catenary risers were also studied by Mazzilli and Sanches [28] using the nonlinear normal mode approach where the fluid forces were modeled using the phenomenological model from [1]. The same approach was later used for straight risers modelling in [29] where the fluid forces were described by IwanBlevins phenomenological model.

Overall, although a number of models describing interactions between the fluid flow and flexible structures have been developed and published, the obtained numerical results are limited and there is still a need for proper multi-mode analysis. Several of the available models only look at the horizontal configuration of the structure, where the pretension is acting in the direction perpendicular to the distributed weight of the structure. The weight distribution becomes important in the vertical configurations, where these forces are acting along the same axis, and therefore this paper will look at the comparison of the multi-mode responses of vertical and horizontal configurations of a pipe.

The rest of the paper is organised as follows. In the next section the mathematical model of the riser system is explained, which is followed by a description of the proposed reduction procedure in Section 3. The responses are analysed in Section 4, where the dependence of the solution on key system parameters is shown and influence of the number of modes retained in the model on the system response is considered. Finally, some concluding remarks are provided in Section 5. 


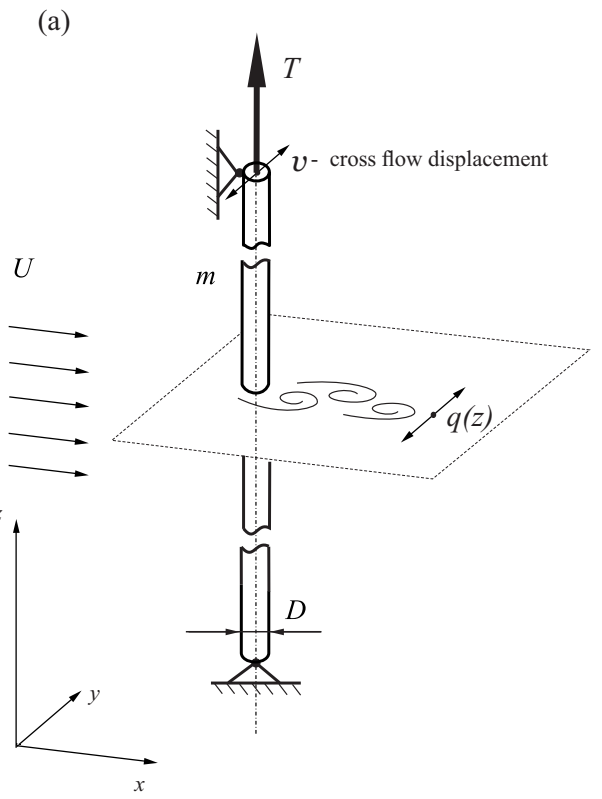

(b)

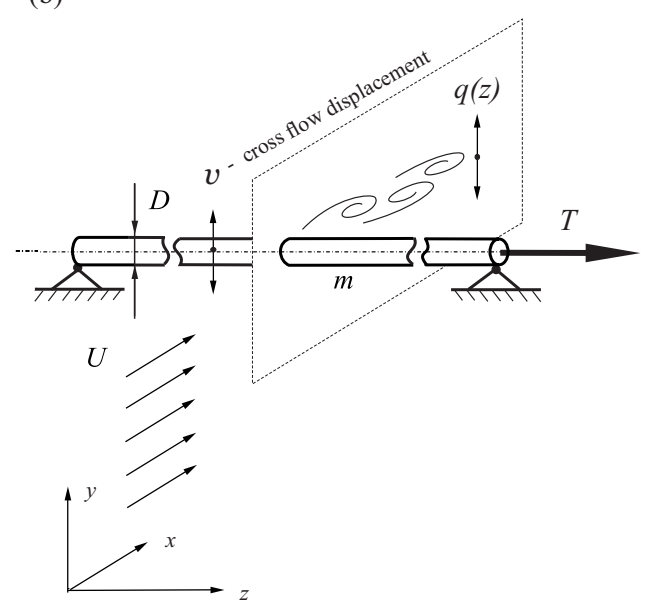

Figure 1: Model of the considered system in (a) vertical and (b) horizontal configurations. The pipe is subject to a uniform flow with velocity $U$ acting in the $x$ direction. Cross-flow displacement of the pipe $v(z, t)$ and non-dimensional wake coefficient $q(z, t)$ are the main unknowns.

\section{Description of the model}

The adopted model for the fluid-structure interactions considered here consists of a straight but slender pipe interacting with uniform flow. Two configurations of the pipe are studied, i.e. vertically and horizontally positioned structures as shown in Fig. 1. The pipes are modelled as an Euler-Bernoulli beam with flexural stiffness. Structural damping can be neglected in the first instance as damping of risers is dominated by hydrodynamic forces and internal damping of the pipe is considered to be very low [30]. The pretension $T$ is applied, and the length-wise mass distribution is included. The structure is assumed to be moving only in the direction normal to flow as shown in Fig. 1 (cross-flow motion) with its in-line motion neglected. Although for some effects including dual resonances [31], taking into account in-line vibrations is crucial, this assumption could be justified for initial analysis as the cross-flow response dominates over the in-line motion in terms of amplitude as shown by experimental investigations [32] on flexible pipes supported at both ends. The analysis presented here could be easily extended to include in-line motion but it would be a subject for another investigation. The equations of motion presented later in this section are developed for the vertical configuration of the riser, where the pretension $T$ and the weight of the riser are acting along the same axis. It is worth noting that for the horizontal configuration the pretension and weight will be perpendicular to each other, resulting in the term with $w_{s t}$ multiplier not being included in the structural equation, Eq. (1). In the examples presented the influence of sagging of the riser is neglected as is the effect of buoyancy.

The governing differential equation for the vertical continuous structure with purely linear characteristics has the following form:

$$
m_{*} \frac{\partial^{2} v(z, t)}{\partial t^{2}}+E I \frac{\partial^{4} v(z, t)}{\partial z^{4}}-T \frac{\partial^{2} v(z, t)}{\partial z^{2}}-w_{s t} \frac{\partial v(z, t)}{\partial z}=F_{F}(z, t),
$$

where $v(z, t)$ is the cross-flow displacement of the structure, $E$ is Young's modulus, $I$ is the area moment of 


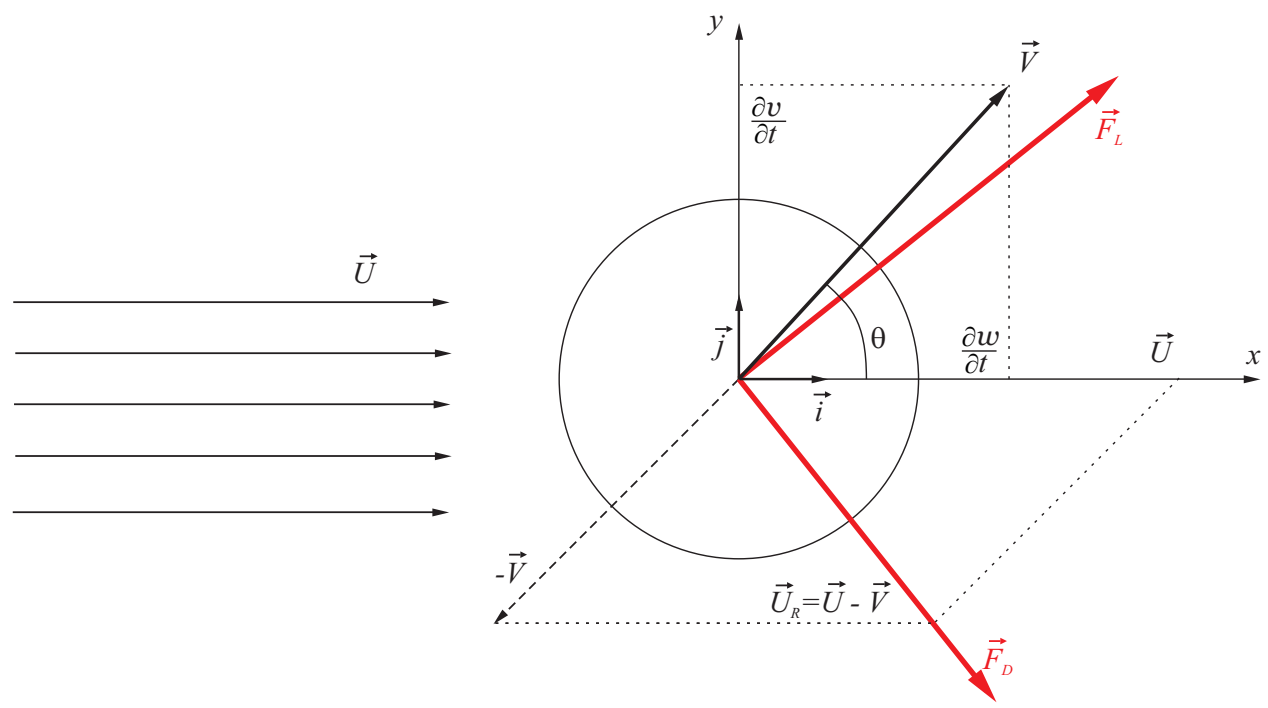

Figure 2: Fluid forces acting on the structure. Drag force $\vec{F}_{D}$ acts in line with relative stream velocity $\vec{U}_{R}$ and lift force $\vec{F}_{L}$ acts in perpendicular direction.

inertia of the pipe, $w_{s t}$ is the weight per unit length and $F_{F}(z, t)$ is the external fluid forcing. The mass per unit of length parameter $m_{*}$ includes the mass of the fluid inside the pipe (for a riser with internal fluid), the mass of the pipe, and the added mass related to the reactive inertial forces from the external fluid:

$$
m_{*}=\rho_{F_{i}} A_{i}+\rho_{S T} A_{S T}+C_{M} \rho_{F_{o}}\left(A_{i}+A_{S T}\right)
$$

where $A_{i}$ and $A_{S T}$ are cross-sectional areas of the pipe bore and pipe wall, and $\rho_{F_{i}}, \rho_{F_{o}}$ and $\rho_{S T}$ are densities of the internal and external fluids and the structure, respectively. The coefficient of added mass $C_{M}$ can be assumed as 1 here since the cross-section of the riser is perfectly circular and the surfaces are ideally smooth [33].

As was discussed in [34], the external fluid force acting on the structure $F_{F}(z, t)$ can be determined as a cross-flow projection of the total hydrodynamic force, $\vec{F}$, which is the result of the actions of the sectional vortex-induced drag $\vec{F}_{D}$ and lift $\vec{F}_{L}$ forces shown in Fig. 2. As can be seen from this figure, the drag force $\vec{F}_{D}$ is acting along the velocity, $\vec{U}_{R}=\vec{U}-\vec{V}$ which is the fluid velocity relative to the cylinder [35] ( $\vec{V}$ is the velocity of the cylinder and $\vec{U}$ is the velocity of the flow). The lift force $\vec{F}_{L}$ is then acting in the perpendicular directions, where the magnitudes of lift and drag forces depend on the magnitude of relative velocity $\vec{U}_{R}$, as discussed in $[36]$.

$$
\begin{aligned}
& \vec{F}_{D}=\frac{1}{2} \rho_{F_{o}} C_{D} D\left|\vec{U}_{R}\right|^{2} \frac{\vec{U}_{R}}{\left|\vec{U}_{R}\right|}, \\
& \vec{F}_{L}=\frac{1}{2} \rho_{F_{o}} C_{L} D\left|\vec{U}_{R}\right|^{2} \mathbf{R} \cdot \frac{\vec{U}_{R}}{\left|\vec{U}_{R}\right|},
\end{aligned}
$$

where $\mathbf{R}=\mathbf{R}\left(\frac{\pi}{2}, \vec{k}\right)$ is the tensor of rotation which rotates the relative velocity vector in a counterclockwise direction by $90^{\circ}$ around axis $\vec{k}$. Using the Rodrigues formulation it can be written as

$$
\mathbf{R}\left(\frac{\pi}{2}, \vec{k}\right)=\cos \left(\frac{\pi}{2}\right)(\mathbf{I}-\vec{k} \otimes \vec{k})+\sin \left(\frac{\pi}{2}\right) \vec{k} \times \mathbf{I}+\vec{k} \otimes \vec{k}=\vec{j} \otimes \vec{i}-\vec{i} \otimes \vec{j}+\vec{k} \otimes \vec{k}
$$

where $\mathbf{I}=\vec{i} \otimes \vec{i}+\vec{j} \otimes \vec{j}+\vec{k} \otimes \vec{k}$ is the unit tensor. Substituting $\vec{U}_{R}=\left(U-\frac{\partial w(z, t)}{\partial t}\right) \vec{i}-\frac{\partial v(z, t)}{\partial t} \vec{j}$ (where $w(z, t)$ is 
in-line displacement) into Eqn.(4), we obtain

$$
\vec{F}_{L}=\frac{1}{2} \rho_{F_{o}} C_{L} D\left|\vec{U}_{R}\right|^{2} \frac{\frac{\partial v(z, t)}{\partial t} \vec{i}+\left(U-\frac{\partial w(z, t)}{\partial t}\right) \vec{j}}{\left|\vec{U}_{R}\right|}
$$

Here the other parameters are $C_{L}$, lift coefficient, and $C_{D}$, total drag. The latter can be represented as a sum of a constant mean sectional drag, $C_{D_{0}}$ and fluctuating drag, $C_{D}^{f l}$

$$
C_{D}=C_{D_{0}}+C_{D}^{f l}
$$

Since in this paper we are limiting our consideration to motion in the cross-flow directions only, we will assume that $\frac{\partial w(z, t)}{\partial t}=0$ and therefore $\vec{U}_{R}=U \vec{i}-\frac{\partial v(z, t)}{\partial t} \vec{j}$, and

$$
\begin{aligned}
\vec{F}_{D} & =\frac{1}{2} \rho_{F_{o}} C_{D} D\left|\vec{U}_{R}\right|\left(U \vec{i}-\frac{\partial v(z, t)}{\partial t} \vec{j}\right), \\
\vec{F}_{L} & =\frac{1}{2} \rho_{F_{o}} C_{L} D\left|\vec{U}_{R}\right|\left(\frac{\partial v(z, t)}{\partial t} \vec{i}+U \vec{j}\right) .
\end{aligned}
$$

Then we can work out the value of total hydrodynamic force component acting in the cross-flow direction $F_{F}(z, t)$ as an appropriate projection of the sum of lift and total drag forces $\vec{F}=\vec{F}_{L}+\vec{F}_{D}$ (see Fig. 2). Therefore we have

$$
F_{F}(z, t)=\left(\vec{F}_{L}+\vec{F}_{D}\right) \cdot \vec{j}=\frac{1}{2} \rho_{F_{o}} C_{L} D\left|\vec{U}_{R}\right| U-\frac{1}{2} \rho_{F_{o}} C_{D} D\left|\vec{U}_{R}\right| \frac{\partial v(z, t)}{\partial t} .
$$

Following the approach of employing nonlinear oscillator equations of the Van der Pol type [15] [16] [24] [36], the fluctuating lift $C_{L}$ and drag $C_{D}^{f l}$ coefficients could be modeled by two wake oscillators [34]. However in this paper we will only consider the mean amplified drag assuming that $C_{D} \approx C_{D_{0}}$ and therefore we will use only one wake oscillator to describe the fluctuating lift $C_{L}=C_{L_{0}} q(z, t) / 2$, where $q(z, t)$ is governed by the following equation

$$
\frac{\partial^{2} q(z, t)}{\partial t^{2}}+\lambda \Omega_{F}\left(q(z, t)^{2}-1\right) \frac{\partial q(z, t)}{\partial t}+\Omega_{F}^{2} q(z, t)=F_{S}(z, t) .
$$

Here $\lambda$ is the Van der Pol parameter, $\Omega_{F}$ is the frequency of vortex shedding, and $F_{S}(z, t)$ is the structural force. Adopting the acceleration coupling as proposed by Facchinetti et al [18], we obtain

$$
F_{S}(z, t)=A \frac{\partial^{2} v(z, t)}{\partial t^{2}}
$$

Finally, assuming that $\left|\frac{\partial v(z, t)}{\partial t}\right| \ll U$ (see [35], for example) and therefore $\left|\vec{U}_{R}\right| \approx U$, we obtain the final expression for the external fluid forces as

$$
F_{F}(z, t)=\frac{1}{4} \rho_{F_{o}} C_{L_{0}} D U^{2} q(z, t)-\frac{1}{2} \rho_{F_{o}} C_{D_{0}} D U \frac{\partial v(z, t)}{\partial t},
$$

which corresponds well to forces used in the widely accepted model [18]. Thus we obtain a system of the coupled equations

$$
\begin{aligned}
& m_{*} \frac{\partial^{2} v(z, t)}{\partial t^{2}}+E I \frac{\partial^{4} v(z, t)}{\partial z^{4}}-T \frac{\partial^{2} v(z, t)}{\partial z^{2}}-w_{s t} \frac{\partial v(z, t)}{\partial z}=\frac{1}{4} \rho_{F_{o}} C_{L_{0}} D U^{2} q(z, t)-\frac{1}{2} \rho_{F_{o}} C_{D_{0}} D U \frac{\partial v(z, t)}{\partial t}, \\
& \frac{\partial^{2} q(z, t)}{\partial t^{2}}+\lambda \Omega_{F}\left(q(z, t)^{2}-1\right) \frac{\partial q(z, t)}{\partial t}+\Omega_{F}^{2} q(z, t)=A \frac{\partial^{2} v(z, t)}{\partial t^{2}} .
\end{aligned}
$$

In order to solve this coupled problem, the Galerkin-type discretisation of transverse $v(z, t)$ displacement and wake coefficient $q(z, t)$ is performed. Details of this reduction process are presented in the following section. 


\section{The reduction procedure}

The decomposition of the system's response into its modal components is achieved through substitution of the Galerking-type discretisation of transverse displacement $v(z, t)$ and wake coefficient $q(z, t)$ into Eqs. (14) and (15) in a similar way as was done in [25]

$$
v(z, t)=\sum_{n=1}^{N} v_{n}(t) \tilde{v}_{n}(z), \quad q(z, t)=\sum_{n=1}^{N} q_{n}(t) \tilde{q}_{n}(z)
$$

Assuming that

$$
\tilde{v}_{n}(z)=\tilde{q}_{n}(z)=\sin \left(n \pi \frac{z}{L}\right)
$$

we have

$$
v(z, t)=\sum_{n=1}^{N} v_{n}(t) \sin \left(n \pi \frac{z}{L}\right), \quad q(z, t)=\sum_{n=1}^{N} q_{n}(t) \sin \left(n \pi \frac{z}{L}\right) .
$$

By invoking the orthogonality property of linear normal modes

$$
\int_{0}^{L} \tilde{v}_{n}(z) \tilde{v}_{i}(z) d z= \begin{cases}0, & n \neq i \\ L / 2, & n=i\end{cases}
$$

and using the following non-dimensional parameters and variables

$$
\omega_{R n}=\frac{\omega_{n}}{\omega_{\text {ref }}} ; \quad \omega_{n}=\frac{\pi n}{L} \sqrt{\frac{E I(\pi n)^{2}}{L^{2} m_{*}}+\frac{T}{m_{*}}}, \quad \Omega_{F}=2 \pi S_{t} \frac{U}{D}, \quad \Omega_{R}=\frac{\Omega_{F}}{\omega_{\text {ref }}} ; \quad \tau=\omega_{\text {ref }} t, \quad \tilde{z}=\frac{z}{L}, \quad \bar{v}=\frac{v}{D},
$$

the following governing equation is obtained for the motion of the linear structure in the $n$-th normal mode (to simplify the formulae the bar sign is omitted below so $v$ is used instead $\bar{v}$ ):

$$
\frac{d^{2} v_{n}(\tau)}{d \tau^{2}}+a \Omega_{R} \frac{d v_{n}(\tau)}{d \tau}+\omega_{R n}^{2} v_{n}(\tau)=b \Omega_{R}^{2} q_{n}(\tau)+\frac{2 w_{s t}}{m_{*} L \omega_{\mathrm{ref}}^{2}} \sum_{i=1}^{N} \Phi_{i n} v_{i}(\tau),
$$

where

$$
\begin{gathered}
\Phi_{i n}=\int_{0}^{1}(\pi i) \cos (\pi i \zeta) \sin (\pi n \zeta) d \zeta, \\
a=\frac{1}{4} \frac{\rho_{F_{o}} C_{D_{0}} D^{2}}{\pi S_{t} m_{*}}, \quad b=\frac{1}{16} \frac{\rho_{F_{o}} C_{L_{0}} D^{2}}{\pi^{2} S_{t}^{2} m_{*}} .
\end{gathered}
$$

$\omega_{\text {ref }}$ is the reference frequency and the $n$-th non-dimensional transverse natural frequency is denoted as $\omega_{R n}$. The dynamical characteristics are now described in the temporal domain while the spatial distribution of the response $\tilde{v}_{n}(z)$ is defined by a harmonic function that satisfies the boundary conditions,

$$
\begin{aligned}
& v(0, t)=0, \quad v(L, t)=0, \\
& \frac{d^{2} v}{d z^{2}}(0, t)=0, \quad \frac{d^{2} v}{d z^{2}}(L, t)=0 .
\end{aligned}
$$

The equation for wake coefficient is transformed in a similar manner and the following results are obtained for the $n$-th component

$$
\frac{d^{2} q_{n}(\tau)}{d \tau^{2}}-\lambda \Omega_{R} \frac{d q_{n}(\tau)}{d \tau}+\lambda \Omega_{R} \sum_{i=1}^{N} \sum_{k=1}^{N} \sum_{s=1}^{N}\left(\Psi_{i k s n} \dot{q}_{i}(\tau) q_{k}(\tau) q_{s}(\tau)\right)+\Omega_{R}^{2} q_{n}(\tau)=A \frac{d^{2} v_{n}(\tau)}{d \tau^{2}}
$$


where

$$
\Psi_{i k s n}=\int_{0}^{1} \sin (\pi i \zeta) \sin (\pi k \zeta) \sin (\pi s \zeta) \sin (\pi n \zeta) d \zeta .
$$

Therefore, in order to calculate the system response as $N$ mode approximation, the coupled system of ordinary differential equations Eqs. $(20,21)$ and $(24,25)$ needs to be solved. Then the obtained functions of time $v_{n}(\tau)$ and $q_{n}(\tau)$ should be substituted into Eq. (16) to determine the system behaviour both in time and along the structure in terms of displacement $v(\tilde{z}, \tau)$ and wake coefficient $q(\tilde{z}, \tau)$.

Thus we obtain the set of equations for one mode approximation which are

$$
\begin{aligned}
& \frac{d^{2} v_{1}(\tau)}{d \tau^{2}}+a \Omega_{R} \frac{d v_{1}(\tau)}{d \tau}+\omega_{R 1}^{2} v_{1}(\tau)=b \Omega_{R}^{2} q_{1}(\tau), \\
& \frac{d^{2} q_{1}(\tau)}{d \tau^{2}}+\lambda \Omega_{R}\left(q_{1}(\tau)^{2}-1\right) \frac{d q_{1}(\tau)}{d \tau}+\Omega_{R}^{2} q_{1}(\tau)=A \frac{d^{2} v_{1}(\tau)}{d \tau^{2}} .
\end{aligned}
$$

In the case of one mode approximation the displacement and wake coefficient along the structure could be calculated as

$$
\begin{aligned}
& v(\tilde{z}, \tau)=v_{1}(\tau) \tilde{v}_{1}(\tilde{z})=v_{1}(\tau) \sin (\pi \tilde{z}), \\
& q(\tilde{z}, \tau)=q_{1}(\tau) \sin (\pi \tilde{z}) .
\end{aligned}
$$

For two-mode approximation, the equations are

$$
\begin{aligned}
& \frac{d^{2} v_{1}(\tau)}{d \tau^{2}}+a \Omega_{R} \frac{d v_{1}(\tau)}{d \tau}+\omega_{R 1}^{2} v_{1}(\tau)=b \Omega_{R}^{2} q_{1}(\tau)+\frac{2 m_{s t} g}{m_{*} L \omega_{\mathrm{ref}}^{2}} \Phi_{21} v_{2}(\tau), \\
& \frac{d^{2} v_{2}(\tau)}{d \tau^{2}}+a \Omega_{R} \frac{d v_{2}(\tau)}{d \tau}+\omega_{R 2}^{2} v_{2}(\tau)=b \Omega_{R}^{2} q_{2}(\tau)+\frac{2 m_{s t} g}{m_{*} L \omega_{\mathrm{ref}}^{2}} \Phi_{12} v_{1}(\tau), \\
& \frac{d^{2} q_{1}(\tau)}{d \tau^{2}}+\lambda \Omega_{R}\left(\frac{3}{4} q_{1}(\tau)^{2} \frac{d q_{1}(\tau)}{d \tau}+\frac{1}{2} q_{2}(\tau)^{2} \frac{d q_{1}(\tau)}{d \tau}+q_{1}(\tau) q_{2}(\tau) \frac{d q_{2}(\tau)}{d \tau}-\frac{d q_{1}(\tau)}{d \tau}\right)+\Omega_{R}^{2} q_{1}(\tau)=A \frac{d^{2} v_{1}(\tau)}{d \tau^{2}}, \\
& \frac{d^{2} q_{2}(\tau)}{d \tau^{2}}+\lambda \Omega_{R}\left(\frac{3}{4} q_{2}(\tau)^{2} \frac{d q_{2}(\tau)}{d \tau}+\frac{1}{2} q_{1}(\tau)^{2} \frac{d q_{2}(\tau)}{d \tau}+q_{1}(\tau) q_{2}(\tau) \frac{d q_{1}(\tau)}{d \tau}-\frac{d q_{2}(\tau)}{d \tau}\right)+\Omega_{R}^{2} q_{2}(\tau)=A \frac{d^{2} v_{2}(\tau)}{d \tau^{2}},
\end{aligned}
$$

and the displacement and wake coefficient along the structure need to be calculated taking both modes into account as

$$
\begin{aligned}
& v(\tilde{z}, \tau)=v_{1}(\tau) \tilde{v}_{1}(\tilde{z})+v_{2}(\tau) \tilde{v}_{2}(\tilde{z})=v_{1}(\tau) \sin (\pi \tilde{z})+v_{2}(\tau) \sin (2 \pi \tilde{z}), \\
& q(\tilde{z}, \tau)=q_{1}(\tau) \sin (\pi \tilde{z})+q_{2}(\tau) \sin (2 \pi \tilde{z}) .
\end{aligned}
$$

The complete set of equations for the three mode approximation is given in the Appendix.

\section{Results}

The results presented in this section are calculated numerically for the set of reference parameters given in Table 1, where the reference frequency $\omega_{\text {ref }}$ was selected as the first natural frequency of the reference structure $\omega_{\text {ref }}=\frac{\pi}{L} \sqrt{\frac{E I \pi^{2}}{L^{2} m_{*}}+\frac{T}{m_{*}}}$. The values of coefficients $C_{L_{0}}, C_{D_{0}}$ and $S_{t}$ were adopted from [1] and for wake oscillator coefficients $\lambda$ and $A$ values similar to those used in [18] were chosen. The system of the developed ordinary differential equations (Eqs.(20), (24)) was solved using an in-house code implemented in FORTRAN and the standard subroutine using Adams-Moulton's method was selected to obtain the solution. 
Table 1: Reference system parameters values

\begin{tabular}{|c|c||c|c|}
\hline Parameter & value & Parameter & Value \\
\hline External Diameter, $D_{o}$ & $0.250 \mathrm{~m}$ & Density of the structure, $\rho_{S T}$ & $7850 \mathrm{~kg} / \mathrm{m}^{3}$ \\
Internal Diameter, $D_{i}$ & $0.125 \mathrm{~m}$ & Density of the water, $\rho_{F_{o}}$ & $1020 \mathrm{~kg} / \mathrm{m}^{3}$ \\
Length, $L$ & $250 \mathrm{~m}$ & Coefficient $C_{D_{0}}$ & 2.0 \\
Young's Modulus, $E$ & $2.10 \cdot 10^{11} \mathrm{~Pa}$ & Coefficient $C_{L_{0}}$ & 0.3 \\
Pretension $T$ & $700000 \mathrm{~N} / \mathrm{m}$ & Coefficient $S_{t}$ & 0.2 \\
Coefficient $\lambda$ & 0.3 & Coefficient $A$ & 10 \\
\hline Reference frequency, $\omega_{\text {ref }}$ & $\frac{\pi}{L} \sqrt{\frac{E I(\pi)^{2}}{L^{2} m_{*}}+\frac{T}{m_{*}}}=0.57174 \mathrm{rad} / \mathrm{s}$ \\
\hline
\end{tabular}

First we compare the responses of the structure at different locations along the pipe subjected to uniform flows of different velocities. The results are shown for horizontal and vertical configurations in Figs. 3 and 4 , respectively.

For the horizontal configuration, the resonance curves are shown in Fig. 3 at four different points, $\tilde{z}=0.2$, 0.25, 0.50 and 0.75 . The graphs presented in parts (a)-(d) demonstrate the dependence of the steady state response of the structure in terms of cross-flow displacement amplitude on the frequency ratio, $\Omega_{R}=\Omega_{F} / \omega_{\text {ref }}$. As the frequency ratio $\Omega_{R}$ is proportional to the external flow velocity, $U$ as $\Omega_{R}=2 \pi S_{t} \frac{U}{D} / \omega_{\text {ref }}$, and the solution is obtained based on a four-mode approximation, the first four resonance peaks are observed under variation of the flow velocity. It is worth noting that the same response is observed for $\tilde{z}=0.25$ and 0.75 in this case as the weight of the structure is acting in perpendicular direction to the pretension, $T$, resulting in the symmetrical response of the structure. As can be seen from Figs. 3(b) and (d), zero solution is observed for $\tilde{z}=0.25$ and 0.75 around fourth resonance $\Omega_{R} \in(3.71,5.48)$ near the node of the fourth mode $\tilde{v}_{4}(\tilde{z})=\sin (4 \pi \tilde{z})$. Fig. 3(c) shows that for $\tilde{z}=0.50$, there are two regions of zero solutions, i.e. $\Omega_{R} \in(1.61,2.62)$ around the second resonance and $\Omega_{R} \in(3.71,5.48)$ around the fourth resonance near the nodes of the second mode $\tilde{v}_{2}(\tilde{z})=\sin (2 \pi \tilde{z})$. The obtained zero solutions demonstrate that although the system of equations for four-mode approximation is nonlinear and all variables are coupled through the relevant wake equations, in this case there is little influence of the structural modes on each other. Also it can be seen from Figs. 3(a)-(d) that the highest vibration amplitude for all locations is approximately at the same level, although the maximum value varies for different flow velocities and for different coordinates.

The dependence of the steady state response of the structure in terms of cross-flow displacement amplitude on the frequency ratio, $\Omega_{R}=\Omega_{F} / \omega_{\text {ref }}$ is demonstrated in Fig. 4 for the vertical configuration. Here the solution is based on five-mode approximation and the resonance curves are presented at the locations $\tilde{z}=0.20,0.25,0.33$, 0.50, 0.70 and 0.90 with the first five resonance peaks observed. In this case the weight of the structure is acting along the same direction as the pretension, $T$ and therefore the structure's response is no longer symmetrical. As can be seen from Fig. 4 the overall maximum of the cross-flow displacement amplitude is decreasing with the increase of the coordinate $\tilde{z}$ as the pretension is being off set by the weight resulting in the higher amplitude of motion in the lower part of the pipe. The lock-in to near zero (low amplitude) solutions is also observed for the vertical configuration of the structure, however these solutions are no longer zero solutions as the additional terms in the right hand side of Eq. (20) introduce direct coupling between the structural modes. As can be seen from Fig. 4(c), the lock-in to low amplitude solution is observed for $\tilde{z}=0.50$ for $\Omega_{R} \in(1.76,2.64)$ around the second resonance and $\Omega_{R} \in(3.73,4.82)$ around the fourth resonance near the nodes of the second mode 
(a)
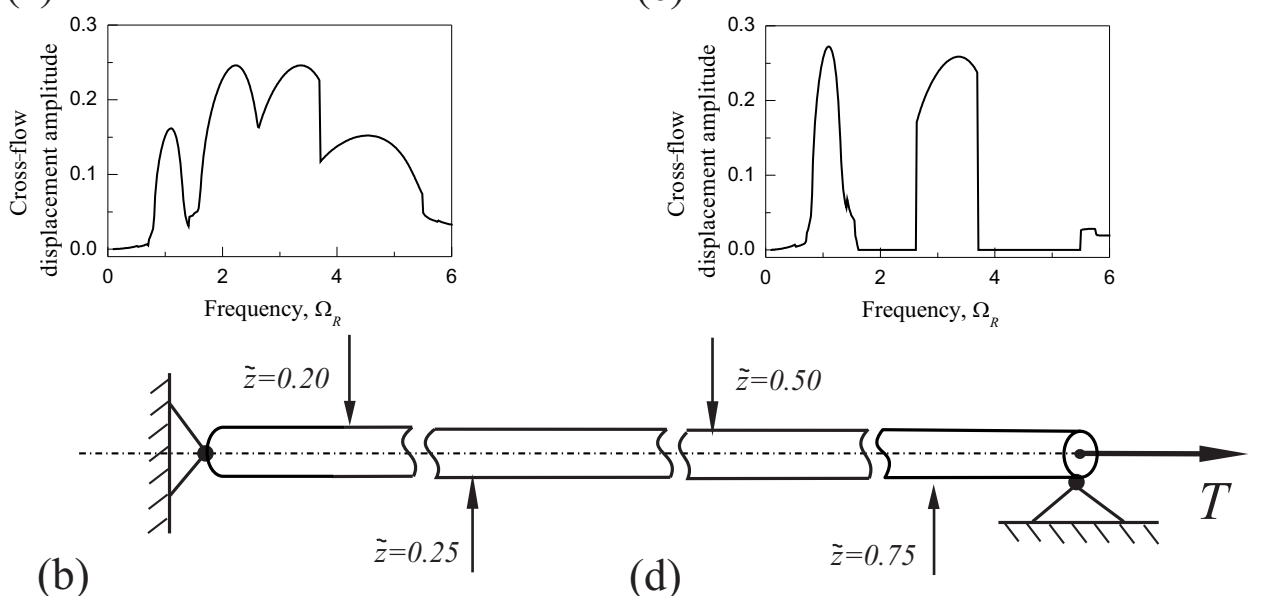

(b)

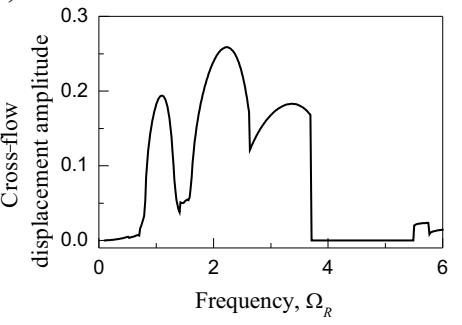

(c)

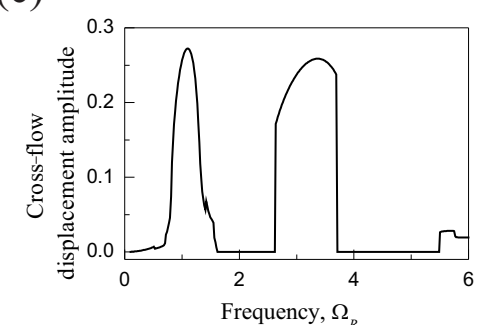

(d)

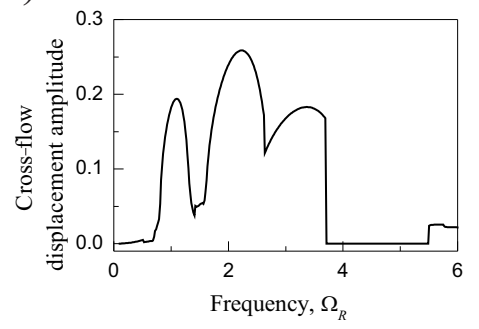

Figure 3: Resonance curves for four-mode approximation at different locations along the horizontal pipe for (a) $\tilde{z}=0.20$, (b) $\tilde{z}=0.25$, (c) $\tilde{z}=0.50$, (d) $\tilde{z}=0.75$.

$\tilde{v}_{2}(\tilde{z})=\sin (2 \pi \tilde{z})$. Fig. $4(\mathrm{~d})$ shows a low amplitude solution around third resonance $\Omega_{R} \in(2.64,3.71)$ near the node of the third mode $\tilde{v}_{3}(\tilde{z})=\sin (3 \pi \tilde{z})$ and Figs. $4(\mathrm{e})$ and $4(\mathrm{f})$ demonstrate lock-in to low amplitude solution around fourth and fifth resonances for $\Omega_{R} \in(3.73,4.84)$ and $\Omega_{R} \in(4.84,6.71)$, respectively. For the coordinate $\tilde{z}=0.70$ which is located between the nodes for mode $\tilde{v}_{3}(\tilde{z})=\sin (3 \pi \tilde{z})$ at $\tilde{z}=0.666667$ and mode $\tilde{v}_{4}(\tilde{z})=\sin (4 \pi \tilde{z})$ at $\tilde{z}=0.75$, the amplitude of the vibrations is reduced near third and fourth resonances at $\Omega_{R} \in(2.64,3.71)$ and $\Omega_{R} \in(3.71,4.82)$, respectively (see Fig. 4(b)).

It is interesting to compare the solutions for vertical and horizontal configurations at the same locations, and Fig. 5 demonstrates such comparison for $\tilde{z}=0.25$ (part a) and $\tilde{z}=0.75$ (part b). The results are presented for three-mode approximation as dependence of the cross-flow amplitude on the frequency $\Omega_{R}$. Here two co-existing solutions were obtained for the horizontal configuration around the second resonance peak at $\Omega_{R} \in(1.44,2.63)$ and they are shown in pink and grey. The vertical configuration solution is given in navy. In general, a higher amplitude of vibration is observed for the vertical configuration in the lower part of the structure, as can be seen from Fig. 5(a) for $\tilde{z}=0.25$, and a lower amplitude is obtained for this configuration in the upper part of the pipe (Fig. 5(b)). As was mentioned before, the horizontal configuration solutions are identical for the chosen coordinates of the pipe due to the symmetry of the structure and constant pretension along it. The vertical configuration solution is affected by the weight distribution and therefore the response is different for $\tilde{z}=0.25$ and 0.75 .

The co-existing solutions for the horizontal configuration are shown in Figs. 6 and 7 for $\tilde{z}=0.25$ and 0.75 , respectively. In parts (a) of these figures, time histories of the steady state response of the system in terms of the cross-flow displacement are presented for $\Omega_{R}=1.8642$, while parts (b) demonstrate the trajectories of the cross-flow displacement on the phase plane. As can be seen from these figures, the obtained responses are 
(a)

(c)
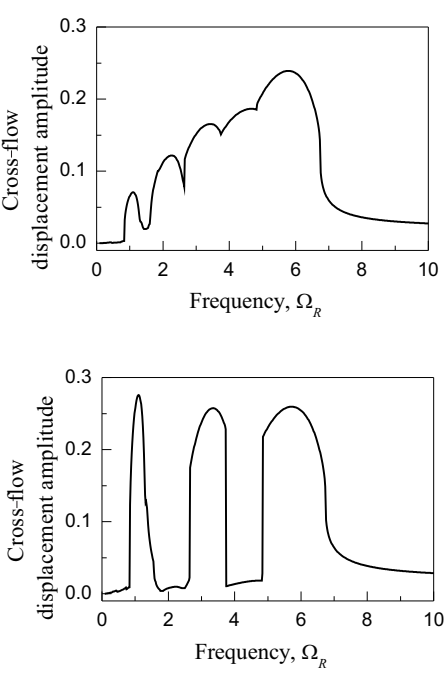

(e)

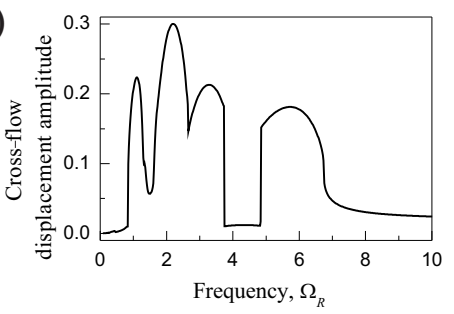

(b)
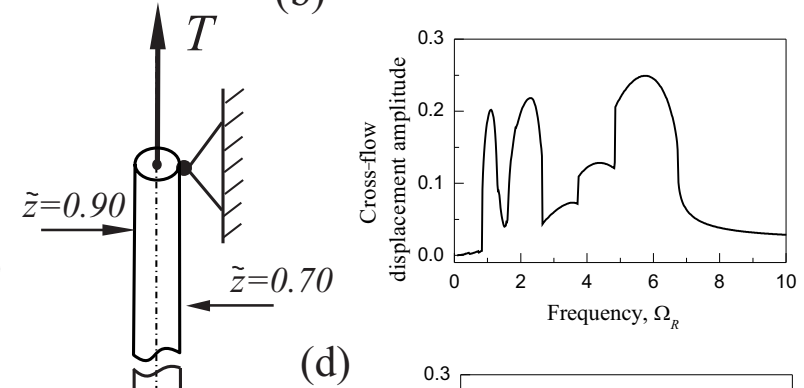

(d)

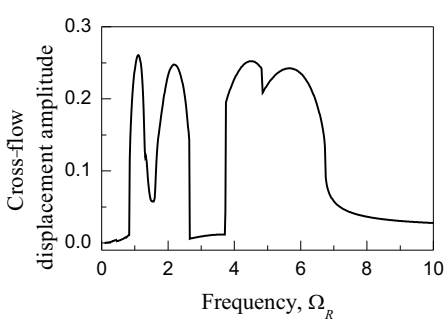

(f)

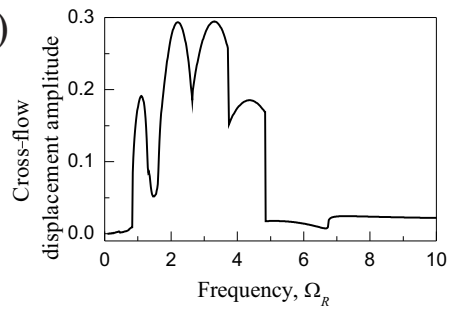

Figure 4: Resonance curves for five-mode approximation at different locations along the riser for (a) $\tilde{z}=0.90,(\mathrm{~b}) \tilde{z}=0.70,(\mathrm{c})$ $\tilde{z}=0.50$, (d) $\tilde{z}=0.33333$, (e) $\tilde{z}=0.25$ and (f) $\tilde{z}=0.20$.

periodic and the non-dimensional frequency of the high amplitude solution (shown in pink) is 1.963 while the frequency of low amplitude solution (shown in grey) is 1.811. For the vertical configuration solution shown in navy at both locations $(\tilde{z}=0.25$ and 0.75$)$, the non-dimensional frequency of the response is 1.963 - the same as for higher amplitude solution obtained for the horizontal configuration. It is worth noting that the non-dimensional frequency of the vortex shedding $\Omega_{R}$ and the frequency of the structure's response do not coincide while the structure is exhibiting the lock-in phenomenon near the second resonance.

Figure 8(a) demonstrates the dependence of the response frequency (for cross-flow vibration) on the frequency ratio $\Omega_{R}=\Omega_{F} / \omega_{\text {ref }}$, which is a function of the flow velocity. Here the dash lines indicate the entrance to and exit from lock-in regions. It can be seen from this figure that the lock-in to the first resonance occurs at $\Omega_{R} \in(0.84,1.25)$ where the frequency of the cross-flow vibration is approximately equal to the first natural frequency of the structure $\left(\omega_{R 1}=\frac{\omega_{1}}{\omega_{\text {ref }}}=1.000\right)$. The second lock-in region is seen for $\Omega_{R} \in(1.62,2.62)$ and here the frequency of the vibration is approximately equal to the second natural frequency $\left(\omega_{R 2}=\frac{\omega_{2}}{\omega_{\text {ref }}}=2.008\right)$. Finally, the third lock-in region is observed for $\Omega_{R} \in(2.62,3.93)$ and in that region the frequency of the vibration is approximately equal to the third natural frequency $\left(\omega_{R 3}=\frac{\omega_{3}}{\omega_{\text {ref }}}=3.032\right)$. The observed slight variation in the response frequency in the lock-in regions might be connected with the current assumption of the constant added mass and it will be investigated in the future. It is interesting to note that as the flow velocity increases there is a break between the first and second lock-in regions, but there is no break between the second and third lock-in 
(a)

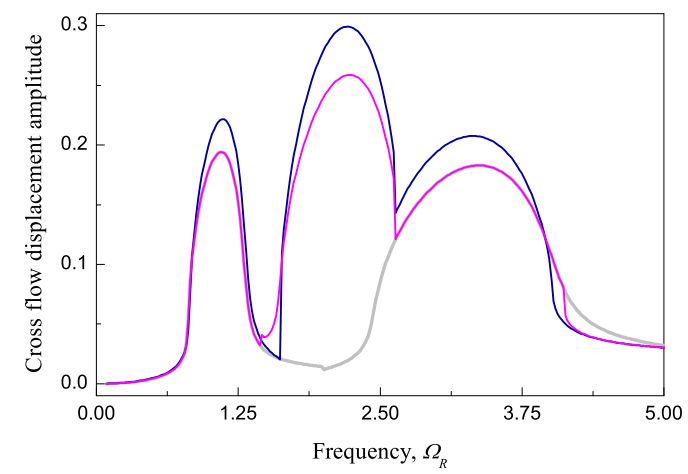

(b)

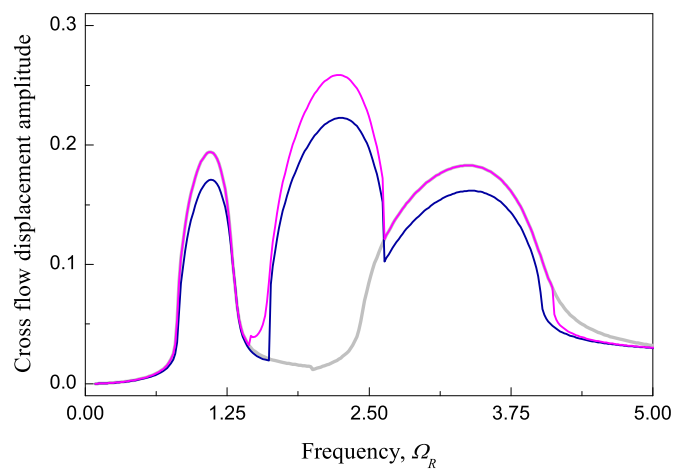

Figure 5: (colour online) Resonance curves for three-mode approximation for different coordinates along the riser for (a) $\tilde{z}=0.25$ and (b) $\tilde{z}=0.75$. The solution for vertical configuration is shown in navy and co-existing solutions for horizontal configuration are presented in pink and grey.

(a)

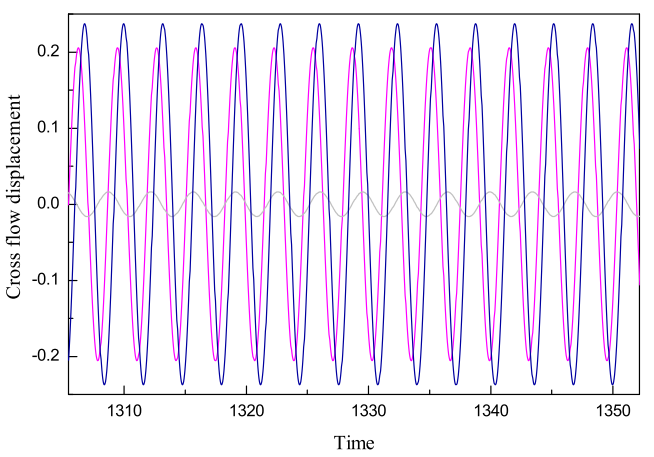

(b)

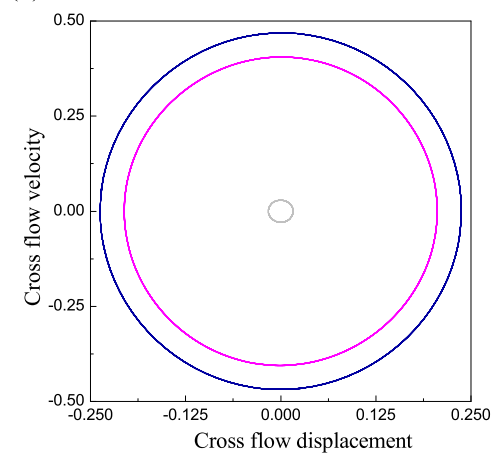

Figure 6: (colour online) (a) Time histories of cross-flow displacement and (b) trajectories on the phase plane for three-mode approximation at $\tilde{z}=0.25$ for $\Omega_{R}=1.8642$. The solution for vertical configuration is shown in navy and co-existing solutions for horizontal configuration are presented in pink and grey.

regions. Figures 8(b)-(e) show how the trajectories of the structure's response on the phase plane change with the frequency ratio. Specifically, Figs. 8(b) and 8(c) show the jump to second lock-in response when frequency ratio changes from $\Omega_{R}=1.58249$ to 1.62645 . Figures $8(\mathrm{~d})$ and $8(\mathrm{e})$ demonstrate the trajectories at the border of the third lock-in region at $\Omega_{R}=3.95623$ and outside it at $\Omega_{R}=4.96726$. There is a notable change not only in the amplitude but also in the shape of the solution.

The influence of the coupling coefficient $A$ and the wake equation coefficient $\lambda$ was analysed next and the results are presented in Fig. 9 for the vertical configuration. Four-mode approximation was used to calculate the presented resonance curves showing the dependence of cross-flow amplitude on non-dimensional vortex shedding frequency, $\Omega_{R}$ at $\tilde{z}=0.4$. In Fig. 9 (a) the results of the calculations are presented for four different values of the coupling coefficient $A=8,10,12$ and 14 and $\lambda=0.3$. As can be seen from this figure, the amplitudes of the vibrations increase significantly in the whole frequency region at the larger values of $A$. Also, a slight shift towards higher frequencies is observed for the maximum amplitude values in each resonant region and the jump between the lock-in solutions becomes larger for higher frequency values.

Fig. 9(b) demonstrates the influence of the coefficient $\lambda$, where four values of this coefficient $\lambda=0.20$, $0.25,0.30$ and 0.35 were considered at $A=10$. As can be seen, increasing $\lambda$ value leads to a decrease of the amplitudes of vibration, and there are no observable frequency shifts for the local maximums. Similar results 
(a)

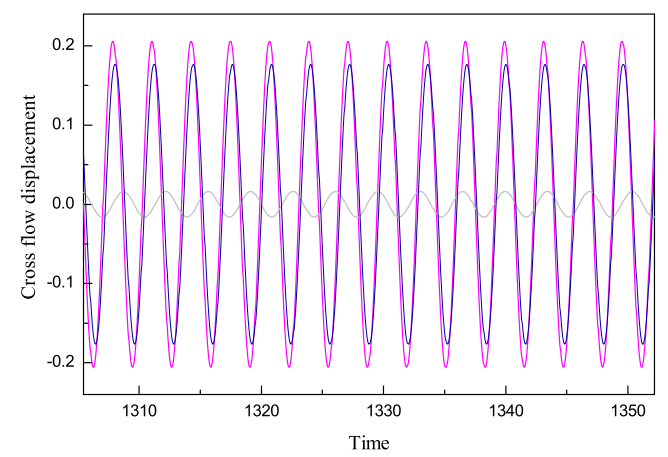

(b)

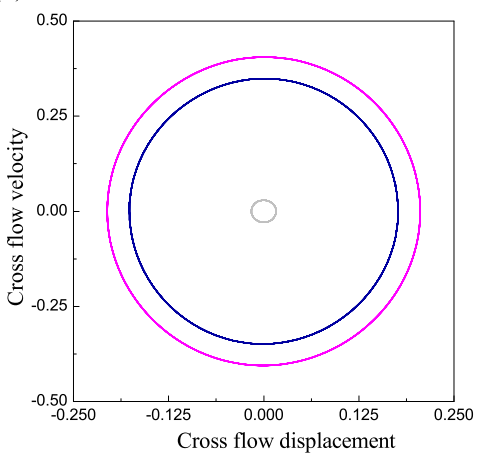

Figure 7: (colour online) (a) Time histories of cross-flow displacement and (b) trajectories on the phase plane for three-mode approximation at $\tilde{z}=0.75$ for $\Omega_{R}=1.8642$. The solution for vertical configuration is shown in navy and co-existing solutions for horizontal configuration are presented in pink and grey.

(a)

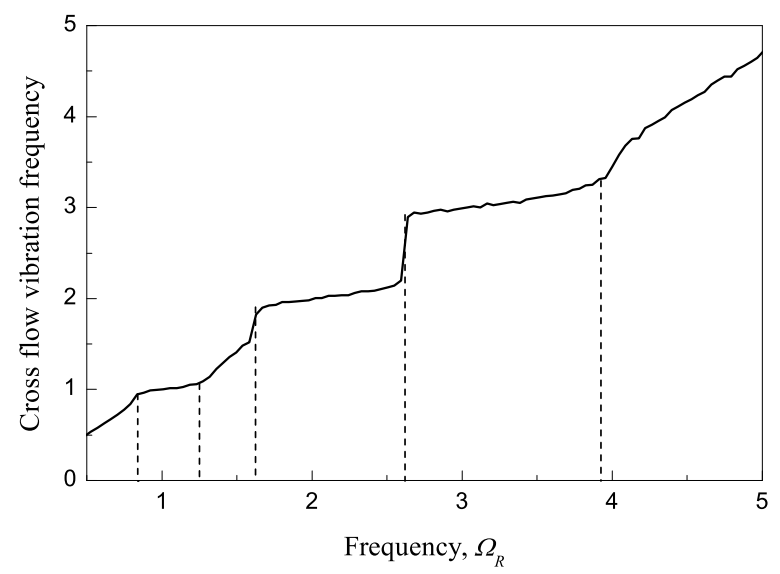

(b)

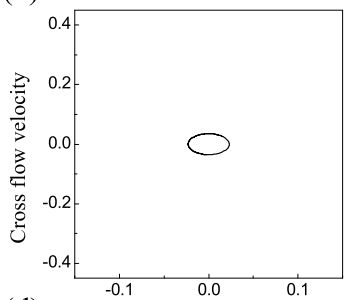

(d)

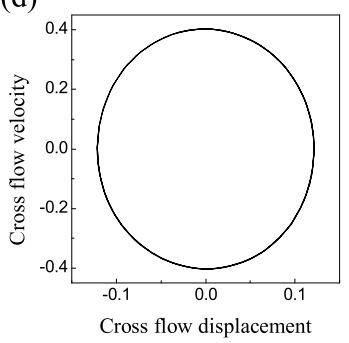

(c)

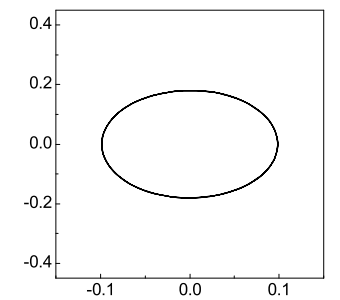

(e)

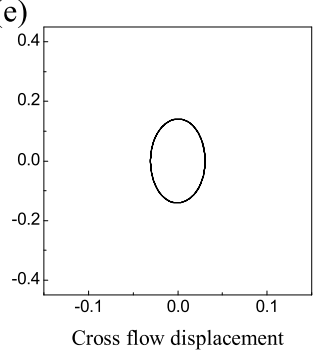

Figure 8: (a) Frequency of the cross-flow vibration for vertical configuration as function of $\Omega_{R}$ for three-mode approximation at $\tilde{z}=0.25$ and trajectories on the phase plane for (b) $\Omega_{R}=1.58249$; (c) $\Omega_{R}=1.62645$; (d) $\Omega_{R}=3.95623$ and (e) $\Omega_{R}=4.96726$. The dash lines in part (a) indicate entrance to and exit from lock-in.

are also obtained for the horizontal configuration for both $A$ and $\lambda$ coefficients but they are not included to avoid repetition.

The other important coefficients influencing the solution are the fluid force coefficients $C_{D_{0}}$ and $C_{L_{0}}$, which are examined in Fig. 10. Fig. 10(a) presents the resonance curves for four different values of the coefficient $C_{D_{0}}=1.6,1.8,2.0$ and 2.2, and Fig. 10(b) shows the results for $C_{L_{0}}=0.25,0.28,0.30$ and 0.33 . As can be seen from these figures obtained for the vertical configuration, increasing the coefficient $C_{D_{0}}$ results in lower amplitudes of vibrations while increasing the coefficient $C_{L_{0}}$ leads to the higher amplitudes. However, as was the case with the wake equation coefficient $\lambda$, neither $C_{D_{0}}$ nor $C_{L_{0}}$ noticeably influences the location of the local maximums along the frequency axis.

The geometry and loading of the structure were also briefly looked at, and Fig. 11 shows the results of the simulations for varying pretension, $T$, and different lengths, $L$ of the vertical pipe. Fig. 11(a) presents the resonance curves for $T=300,400,700$ and $1000 \mathrm{kN}$ calculated for $L=250 \mathrm{~m}$ and Fig. 11(b) demonstrates 
(a)

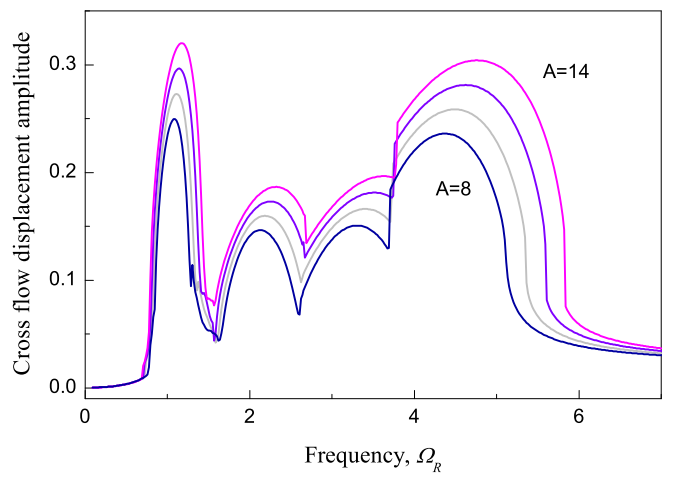

(b)

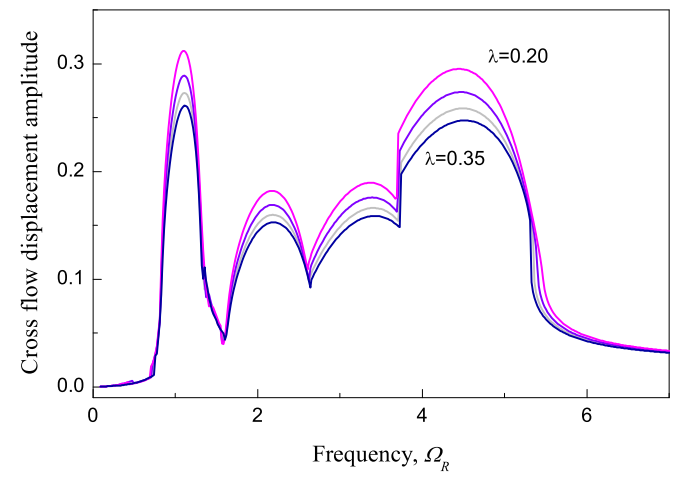

Figure 9: (colour online) Resonance curves for four-mode approximation at $\tilde{z}=0.4$ for different values of (a) coupling coefficient $A=8,10,12$ and 14 and $\lambda=0.3$; and (b) wake equation coefficient $\lambda=0.20,0.25,0.30$ and 0.35 and $A=10$.

(a)

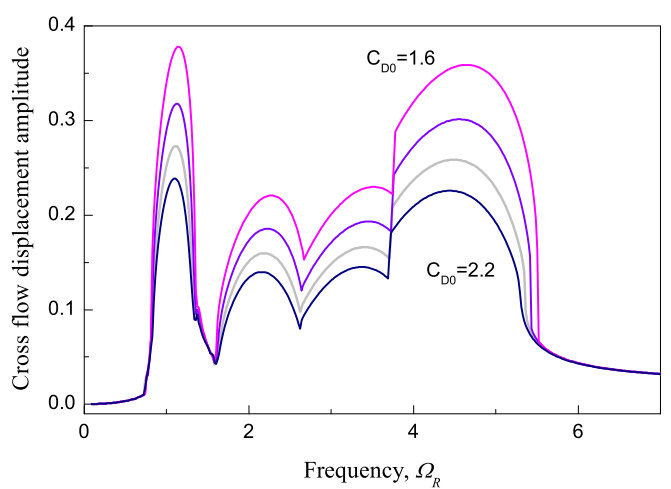

(b)

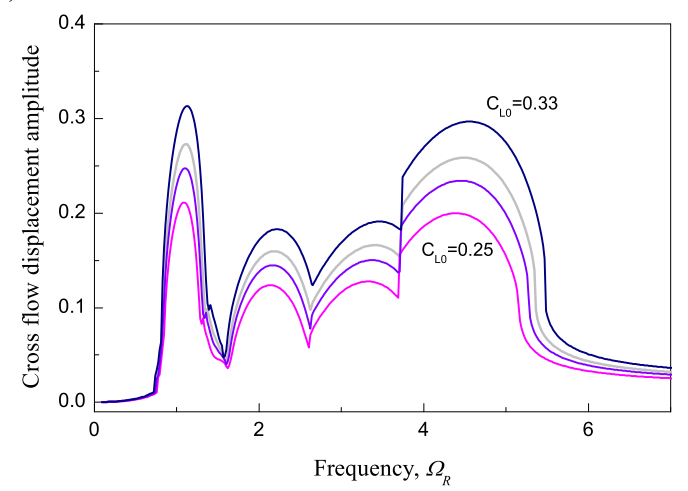

Figure 10: (colour online) Resonance curves for four-mode approximation at $\tilde{z}=0.4$ for different values of (a) coefficient $C_{D_{0}}=2.2$ (in navy), 2.0 (in grey), 1.8 (in purple) and 1.6 (in pink) and (b) coefficient $C_{L_{0}}=0.33$ (in navy), 0.3 (in gray), 0.28 (in purple) and 0.25 (in pink).

cross-flow amplitudes for $L=150,200,250$ and $300 \mathrm{~m}$ obtained at $T=700 \mathrm{kN}$. As can be observed, the resonant frequencies of the structure depends both on pretension, $T$ and length, $L$, and each local peak shifts to the right as pretension is increased or length is decreased. The maximum amplitudes in each resonance region slightly decrease for higher values of pretension and slightly increase for longer pipes, where each lock-in region becomes wider for higher pretension and shorter pipe length.

Finally, Fig. 12 demonstrates the resonance curves for higher mode approximations. The results for $\tilde{z}=0.25$ and $\tilde{z}=0.50$ are shown in Fig. 12(a) and 12(b) respectively for 6 mode (in navy), 7 mode (in pink), 8 mode (in grey) and 9 mode (in purple) approximations. As can be seen from these figures, in all cases each consecutive approximation predicts shorter regions for the penultimate resonance, i.e. for $\tilde{z}=0.25$ the sixth resonant region is observed for $\Omega_{R} \in(5,98,8.18)$ for the six mode approximation (navy line), but the lock-in region is predicted at $\Omega_{R} \in(5.98,7.22)$ for all higher mode approximations. In general, the level of vibrations (peak resonant amplitudes) at lower frequencies is predicted reasonably well by approximations with fewer modes taken into account. However this does not always apply to the last resonant region as the difference in the amplitudes could be substantial, as demonstrated in Fig. 12(a) for six (in navy) and seven (and higher) mode approximations in the sixth resonant region $\Omega_{R} \in(5.98,7.22)$. 
(a)

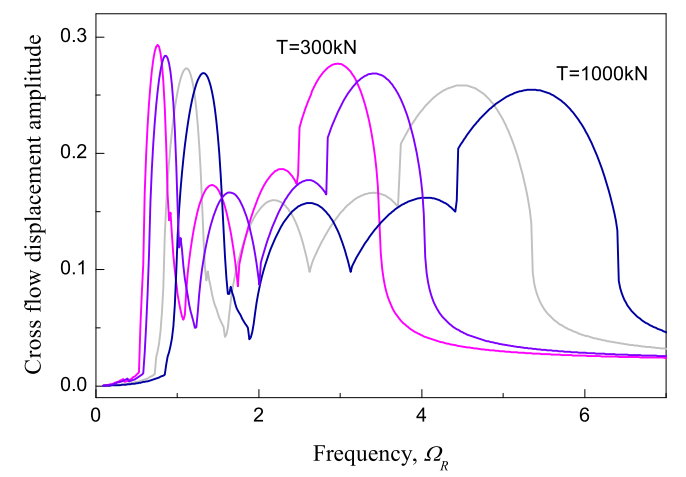

(b)

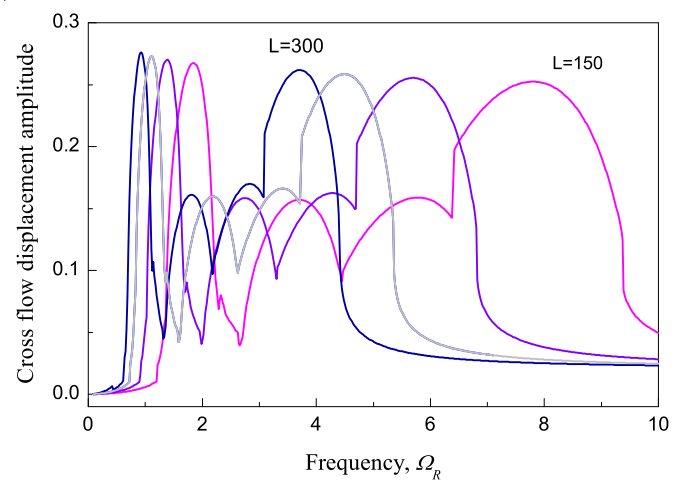

Figure 11: (colour online) Resonance curves for four-mode approximation at $\tilde{z}=0.4$ for different values of (a) tension $T=$ $300,400,700$ and $1000 \mathrm{kN}$ calculated for $L=250 \mathrm{~m}$ and (b) the riser length $L=150 \mathrm{~m}$ (in pink), $200 \mathrm{~m}$ (in purple), $250 \mathrm{~m}$ (in grey) and $300 \mathrm{~m}$ (in navy) at $T=700 \mathrm{kN}$.

(a)

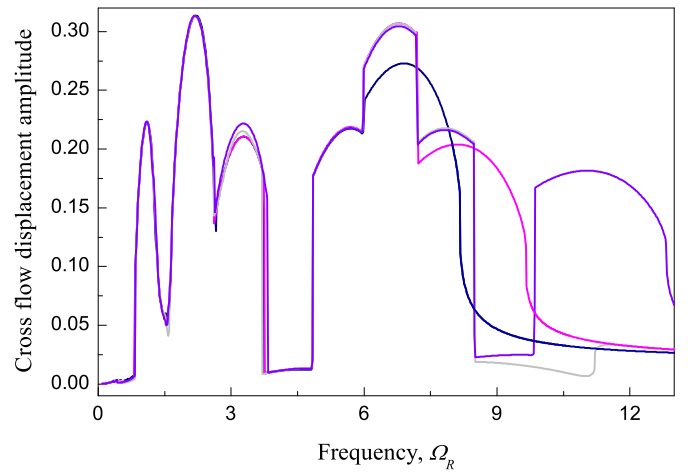

(b)

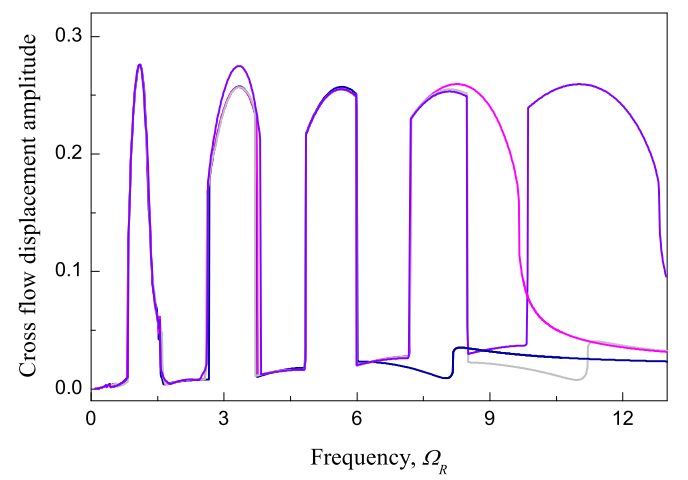

Figure 12: (colour online) Resonance curves for 6 mode (in navy), 7 mode (in pink), 8 mode (in grey) and 9 mode (in purple) approximations for different coordinates along the riser for (a) $\tilde{z}=0.25$ and (b) $\tilde{z}=0.50$. 


\section{Conclusions}

In this work the fluid-structure interactions were considered by investigating a straight but slender pipe interacting with uniform water flow. Two configurations were studied, namely vertically and horizontally positioned pipes, which are modelled as an Euler-Bernoulli beam with flexural stiffness. Both the pretension and the length-wise mass distribution were considered. The structure was assumed to be moving only in the direction normal to flow (cross-flow motion) hence its in-line motion was neglected. The external fluid force acting on the structure is the result of the action of sectional vortex-induced drag and lift forces. Only mean drag was considered, with the time varying lift force modelled by a nonlinear oscillator equation of the Van der Pol type to calculate the non-dimensional lift coefficient. The obtained coupled system of nonlinear partial differential equations was simplified using Galerkin-type discretisation along the pipe. The resulting ordinary differential equations were solved numerically providing multi-mode approximations of cross-flow displacement and non-dimensional lift coefficient.

A comparison between the responses of vertical and horizontal structures has been made. In the case of the horizontal configuration, although the system of equations is nonlinear and all variables are coupled through the relevant wake equations, the structural modes are observed to have little influence on each other. For the vertical configuration however, there is a direct coupling between the structural modes, which is reflected in the shape of the resonance curves. In general, a higher amplitude of vibration is observed for the vertical configuration in the lower part of the structure, and a lower amplitude is obtained in the upper part due to a balancing between pretension and weight. Meanwhile, the horizontal configuration solutions are identical in symmetrical locations along the pipe due to constant pretension.

When studying the influence of the coupling coefficient $A$ (which appears in the wake equation as the multiplier in front of the structural acceleration), it was observed that the amplitudes of the vibrations increased significantly in the whole frequency region at the larger values of this coefficient, and there was a slight shift towards higher frequencies for maximum amplitude values in each of the resonant regions. Our analysis also shows that increasing the value of the wake oscillator coefficient $\lambda$ leads, as expected, to a decrease of the amplitudes of the vibrations, and there are no observable frequency shifts for the local maximums.

The other important coefficients influencing the solution are the fluid force coefficients $C_{D_{0}}$ and $C_{L_{0}}$. It is shown that increasing the coefficient $C_{D_{0}}$ results in lower amplitudes of vibrations while increasing the coefficient $C_{L_{0}}$ leads to higher amplitudes. However, as was the case with wake equation coefficient $\lambda$, neither $C_{D_{0}}$ nor $C_{L_{0}}$ noticeably influences the locations of the local maximums along the frequency axis.

While looking at the geometry and loading of the structure, it was observed that the resonant frequencies of the structure depend both on the pretension, $T$, and the length, $L$, where each of the local peaks shifts to higher values of frequencies as the pretension is increased or length is decreased. The maximum amplitudes in each resonant region slightly decrease for higher values of pretension and slightly increase for longer pipes, where each lock-in regions becomes wider for higher pretension and shorter pipe length.

The resonance curves for higher mode approximations were also studied and in all cases it was observed that each consecutive approximation predicts a shorter lock-in region for the penultimate resonance. In general, the level of vibrations (peak resonant amplitudes) at lower frequencies is predicted reasonably well by approximations with fewer modes taken into account. However, this does not always apply to the last resonant region, as it was shown that the difference in amplitudes could be substantial. In practical VIVs, low frequency modes are of particular interest, therefore, they should be well defined. These results show that in order to achieve better 
accuracy of the highest mode of interest, at least a small set of higher modes, superior to those of interest, should be retained in the model.

Finally, it has to be emphasized that the proper calibration of the proposed model based on the experimental tests will be an important part of future work. Also, the in-line component of the motion will be taken into account in order to improve the model in further investigations.

\section{ACKNOWLEDGMENTS}

AP would like to acknowledge the support of the National Subsea Research Institute (NSRI) UK. EP and MW are grateful for partial support provided by the Italian Ministry of Education, University and Research (MIUR) by the PRIN funded program 2010/11 N.2010MBJK5B.

\section{References}

[1] Blevins, R.D., 1990, "Flow-Induced Vibration", Van Nostrand Reinhold, New York.

[2] Païdouissis, M.P., Price, S.J., and de Langre, E., 2010, "Fluid-Structure Interactions - Cross-Flow Induced Instabilities", Cambridge University Press, New York.

[3] MIT Research: Vortex-Induced Vibration, Department of Ocean Engineering, "SHEAR 7 Simulation", http://web.mit.edu/shear7/shear7.html

[4] Orcaflex software web site, Orcina Ltd., http://www.orcina.com/SoftwareProducts/OrcaFlex/index.php (accessed on 11 February 2015).

[5] Willden, R.H.J., Graham, J.M.R., 2001, "Numerical prediction of VIV on long flexible circular cylinders", European Journal of Mechanics B/Fluids 23, 209-218.

[6] Willden, R.H.J., 2003,"Numerical Prediction of the Vortex-Induced Vibrations of Marine Riser Pipes", PhD Thesis, Department of Aeronautics, Imperial College London.

[7] Willden, R.H.J., Graham, J.M.R., 2004, "Multi-modal Vortex-Induced Vibrations of a vertical riser pipe subject to a uniform current profile", European Journal of Mechanics B/Fluids 23, 209-218.

[8] Yamamoto, C.T., Meneghini, J.R., Saltara, F., Fregonesi, R.A. and Ferrari Jr., J.A., 2004, "Numerical simulations of vortex-induced vibration on flexible cylinders", Journal of Fluids and Structures 19(4), 467489.

[9] Huang, K., Chen, H.-C., Chen, H.-R., 2010, "Vertical Riser VIV Simulation in Uniform Current", Journal of Offshore Mechanics and Arctic Engineering, vol. 132.

[10] Chaplin, J.R., Bearman, P.W., Huera Huarte, F.J., Pattenden, R., 2005, "Laboratory measurements of vortex-induced vibrations of a vertical tension riser in a stepped current", Journal of Fluid and Structures $21,3-24$.

[11] Huera Huarte, F.J., Bearman, P.W., Chaplin, J.R., 2006, "On the force distribution along the axis of a flexible circular cylinder undergoing multi-mode vortex-induced vibrations", Journal of Fluid and Structures 22, 897-903. 
[12] Franzini, G.R., Mazzilli, C.E.N., Pesce, C.P., 2015, "Reduced-order model for parametric excitation of a submerged, vertical and exible cylinder: a calibration approach study", in: DINAME 2015 - Proceedings of the XVII International Symposium on Dynamic Problems of Mechanics, Natal, Brazil, 22-27 February 2015.

[13] Franzini, G.R., Gonalves, R.T., Pesce, C.P., Fujarra, A.L.C., Mazzilli, C.E.N., Meneghini, J.R., Mendes, P., 2015, "Vortex-induced vibration experiments with a long semi-immersed flexible cylinder under tension modulation: Fourier transform and HilbertHuang spectral analyses" Journal of the Brazilian Society of Mechanical Sciences and Engineering, 37(2), pp. 589-599.

[14] Mazzilli, C.E.N., Lenci, S., Demeio, L., 2014, "Non-linear free vibrations of tensioned vertical risers", in: Proceedings of the 8th European Nonlinear Dynamics Conference - ENOC2014, Vienna, Austria, 6-11 July 2014.

[15] Bishop, R.E.D., Hassan, A.Y., 1964, "The Lift and Drag Fores on a Circular Cylinder in a Flowing Field", Proc. Roy. Soc. (London) Ser. A, 277, 51-75.

[16] Skop, R. A., Griffin, O. M., 1973, "A Model for the Vortex-Excited Resonant Vibrations of Bluff Bodies", J. Sound and Vibration 27, 225-233.

[17] Balasubramanian, S., Skop, R.A., 1997, "A New Twist on an Old Model for Vortex-Excited Vibrations", Journal of Fluids and Structures 11, 395-412.

[18] Facchinetti M.L., de Langre E., Biolley F., 2004, "Coupling of Structure and Wake Oscillators in VortexInduced Vibrations", Journal of Fluids and Structures 19, 123-140.

[19] Violette, R., de Langre, E., Szydlowski, J., 2007, "Computation of Vortex-Induced Vibrations of Long Structures Using a Wake Oscillator Model: Comparison with DNS and Experiments", Computers and Structures 85, 1134-1141.

[20] Wang X.Q., So, R.M.C., Chan, K.T. 2003, "A Nonlinear Fluid Force Model for Vortex-Induced Vibration of an Elastic Cylinder", Journal of Sound and Vibration 260, 287-305.

[21] Ge Fei, Long Xu, Wang Lei and Hong YouShi, 2009, "Flow-Induced Vibrations of Long Circular Cylinders Modeled by Coupled Nonlinear Oscillators", Sci China Ser G-Phys Mech Astron, vol. 52, no. 7, $1086-1093$.

[22] Furnes G.K., Sorensen K., 2007, "Flow Induced Vibrations Modeled by Coupled Non-Linear Oscillators", Proceedings of the 17th International Offshore and Polar Engineering Conference, Lisbon, Portugal. 27812787.

[23] da Silveira, L. M. Y., Martins, C. A., Cunha, L. D. and Pesce, C. P., 2007, "An Investigation on the Effect of Tension Variation on VIV of Risers", Proceedings of ASME 2007 26th International Conference on Offshore Mechanics and Arctic Engineering, OMAE2007-29247, pp. 267-275.

[24] Iwan, W.D., Blevins R.D., 1974, "A Model for Vortex-Induced Oscillation of Structures", J. Appl. Mech. $41,581-586$

[25] Keber, M., Wiercigroch, M., 2008, "Dynamics of a Vertical Riser with Weak Structural Nonlinearity Excited by Wakes", Journal of Sound and Vibration 315, 685-699. 
[26] Mazzilli, C.E.N., Sanches, C.T., Baracho Neto, O.G.P., Wiercigroch, M., Keber, M., 2008, "Non-linear modal analysis for beams subjected to axial loads: Analytical and finite-element solutions", International Journal of Non-Linear Mechanics 43(6), 551-561.

[27] Srinil, N., Wiercigroch, M., O'Brien, P., 2009, "Reduced-Order Modelling of Vortex-Induced Vibration of Catenary Riser", Ocean Engineering 36, Issue: 17-18, 1404-1414.

[28] Mazzilli, C.E.N., Sanches, C.T., 2011, "Active Control of Vortex-Induced Vibrations in Offshore Catenary Risers: a Nonlinear Normal Mode Approach", Journal of Mechanics of Materials and Structures 6(7-8), 1079-1088.

[29] Mazzilli, C.E.N., Poncet,R. 2012, "Non-Linear Reduced-Order Modelling of Vortex-Induced Vibrations in Straight Risers", Proceedings of PACAM XII, 12th Pan-American Congress of Applied Mechanics, January 02-06, 2012, Port of Spain, Trinidad.

[30] Assi, G.R.S., Bearman, P.W. and Kitney, N., 2009, "Low Drag Solutions for Suppressing VortexInduced Vibration of Circular Cylinders", Journal of Fluids and Structures 25, 666675.

[31] Dahl, J.M., Hover, F.S., Triantafyllou, M.S., Oakley, O.H., 2010, "Dual resonance in vortex-induced vibrations at subcritical and supercritical Reynolds numbers", Journal of Fluid Mechanics 643, 395-424.

[32] H. Marcollo, J. B. Hinwood, 2006, "On Shear Flow Single Mode Lock-In with Both Cross-Flow and In-Line Lock-In Mechanisms", Journal of Fluids and Structures 22, 197-211.

[33] A. Leonard, A. Roshko, "Aspects of Flow-Induced Vibration", Journal of Fluids and Structures 15 (2001) 415-425.

[34] Pavlovskaia, E., Postnikov, A. and Wiercigroch, M. 2015 "CFD Calibrated Wake Oscillator Model for Vortex-Induced Vibrations", Proceedings of International Conference on Engineering Vibrations, Lujbljana, Slovenia, 7-10 September 2015.

[35] Vandiver, J.K., 1993, "Dimensionless Parameters Important to the Prediction of Vortex-Induced Vibration of Long, Flexible Cylinders in Ocean Currents", Journal of Fluids and Structures 7, 423-455.

[36] Hartlen, R.T., Currie, I.G., 1970, "Lift-Oscillator Model of Vortex-Induced Vibration", Journal of the Engineering Mechanics Division EM5, 577-591.

[37] M. Keber, M. Wiercigroch, "A Reduced Order Model for Vortex-Induced Vibration of a Vertical Offshore Riser in Lock-In", In: Proceedings of the IUTAM Symposium on Fluid-Structure Interaction in Ocean Engineering, Hamburg, Germany (2007)

[38] Vandiver, J.K., Jong, J.-Y., 1987, "The Relationship Between In-Line and Cross-Flow Vortex-Induced Vibration of Cylinders", Journal of Fluids and Structures, 381-399. 


\section{Appendix}

Finally, for three-mode approximation we have

$$
\begin{aligned}
& \frac{d^{2} v_{1}(\tau)}{d \tau^{2}}+a \Omega_{R} \frac{d v_{1}(\tau)}{d \tau}+\omega_{R 1}^{2} v_{1}(\tau)=b \Omega_{R}^{2} q_{1}(\tau)+\frac{2 m_{s t} g}{m_{*} L \omega_{\text {ref }}^{2}} \sum_{i=1}^{3} \Phi_{i 1} v_{i}(\tau) \\
& \frac{d^{2} v_{2}(\tau)}{d \tau^{2}}+a \Omega_{R} \frac{d v_{2}(\tau)}{d \tau}+\omega_{R 2}^{2} v_{2}(\tau)=b \Omega_{R}^{2} q_{2}(\tau)+\frac{2 m_{s t} g}{m_{*} L \omega_{\text {ref }}^{2}} \sum_{i=1}^{3} \Phi_{i 2} v_{i}(\tau) \\
& \frac{d^{2} v_{3}(\tau)}{d \tau^{2}}+a \Omega_{R} \frac{d v_{3}(\tau)}{d \tau}+\omega_{R 3}^{2} v_{3}(\tau)=b \Omega_{R}^{2} q_{3}(\tau)+\frac{2 m_{s t} g}{m_{*} L \omega_{\text {ref }}^{2}} \sum_{i=1}^{3} \Phi_{i 3} v_{i}(\tau) \\
& \frac{d^{2} q_{1}(\tau)}{d \tau^{2}}-\lambda \Omega_{R} \frac{d q_{1}(\tau)}{d \tau}+\lambda \Omega_{R} \sum_{i=1}^{3} \sum_{k=1}^{3} \sum_{s=1}^{3} \Psi_{i k s 1} \dot{q}_{i}(\tau) q_{k}(\tau) q_{s}(\tau)+\Omega_{R}^{2} q_{1}(\tau)=A \frac{d^{2} v_{1}(\tau)}{d \tau^{2}} \\
& \frac{d^{2} q_{2}(\tau)}{d \tau^{2}}-\lambda \Omega_{R} \frac{d q_{2}(\tau)}{d \tau}+\lambda \Omega_{R} \sum_{i=1}^{3} \sum_{k=1}^{3} \sum_{s=1}^{3} \Psi_{i k s 2} \dot{q}_{i}(\tau) q_{k}(\tau) q_{s}(\tau)+\Omega_{R}^{2} q_{2}(\tau)=A \frac{d^{2} v_{2}(\tau)}{d \tau^{2}} \\
& \frac{d^{2} q_{3}(\tau)}{d \tau^{2}}-\lambda \Omega_{R} \frac{d q_{3}(\tau)}{d \tau}+\lambda \Omega_{R} \sum_{i=1}^{3} \sum_{k=1}^{3} \sum_{s=1}^{3}\left(\Psi_{i k s} \dot{q}_{i}(\tau) q_{k}(\tau) q_{s}(\tau)\right)+\Omega_{R}^{2} q_{3}(\tau)=A \frac{d^{2} v_{3}(\tau)}{d \tau^{2}} \\
& \Psi_{i k s j}=\int_{0}^{1} \sin (\pi i \xi) \sin (\pi k \zeta) \sin (\pi s \zeta) \sin (\pi j \zeta) d \xi \\
& \begin{array}{c}
q(\tilde{z}, \tau)=v_{1}(\tau) \tilde{v}_{1}(\tilde{z})+v_{2}(\tau) \tilde{v}_{2}(\tilde{z})+v_{3}(\tau) \tilde{v}_{3}(\tilde{z})=v_{1}(\tau) \sin (\pi \tilde{z})+v_{2}(\tau) \sin (2 \pi \tilde{z})+v_{3}(\tau) \sin (3 \pi \tilde{z}) \\
q_{1}(\tau) \sin (\pi \tilde{z})+q_{2}(\tau) \sin (2 \pi \tilde{z})+q_{3}(\tau) \sin (3 \pi \tilde{z})
\end{array}
\end{aligned}
$$

